\title{
FLORA Y VEGETACIÓN DEL DEPARTAMENTO DE TACNA
}

\author{
Juan Franco Leon ${ }^{1}$; César Cáceres Musaja ${ }^{2}$ Liduvina Sulca Quispe ${ }^{3}$
}

\section{RES U MEN}

El presente artículo es un esiudio de la flora y vegetación del departamento de Tacna, ubicado en el extremo sur occidental del Perú, enmarcado en las coordenadas geográficas $16^{\circ} 59^{\prime}$ y $18^{\circ} 21^{\prime} \mathrm{LS}$. y $69^{\circ} 28^{\prime}$ y $71^{\circ} 10^{\prime} \mathrm{LW}$, en altitudes que van de los 0.0 a $4700 \mathrm{~m}$.

La flora vascular está compuesta de 708 especies, 360 géneros, 101 familias y 4 divisiones, distribuidas en: Pteridophytas (21 especies, representan el 2.97\%), Coniferophyta (3-0.42\%), Gnetophyta (2-0.28\%) y Magnoliophyta (Magnoliopsida, 580-81. 92\% y Liliopsida, (102-14.41\%). Las familias con mayor número de especies son: Asteraceae (151-21.33\%), Poaceae (70-9.89\%), Fabaceae (49-6.92\%), Solanaceae (36-5.08\%) y Malvaceae (29-4.10\%). Del total de las especies 92 son endémicas y 36 solo se reportan para el departamento.

En base a la relación entre geomorfología, litología, altitud, pisos bioclimáticos, intervalos de precipitación, descripción bioclimática, y la composición floristica en determinadas zonas, fueron diferenciadas 17 comunidades vegetales en cuatro zonas muy marcadas. Predominando la vegetación de lomas, tillandsial, monte ribereño, matorral, pajonal, semi desierto, piso de cactaceas, tolares y bosques de queñoales.

Se hace un aporte con respecto a la flora y vegetación existente en el departamento de Tacna agrupándolos en cuanto al claro predominio de algunas especies y al aspecto ecológico del medio donde se desarrollan las especies, se pone en consideración las especies más representativas que se encuentran en las diferentes comunidades vegetales.

\section{$A B S T R A C ?$}

The present article is a study of the flora and egetation of Tacna department, once was located in the southern extreme occidental of the Peru, framed in the geographic coordinates $16^{\circ} 59$ and $18^{\circ} 21^{\prime} \mathrm{LS}$. and $69^{\circ} 28$ and $71^{\circ} 10^{\prime} \mathrm{LW}$, in altitudes that they go from the 0.0 to $4700 \mathrm{~m}$.

The vascular flora this one, repaired 708 species, 360 genera, 101 families and 4 divisions, distributed in: Pteridophytas, (21 species, represents the 2.97\%), Coniferophyta, (3-0.42\%), Gnetophyta (2-0.28\%) and Magnoliophyta (Magnoliopsida, 580-81. 92\% y Liliopsida, (102-14.41\%). The families with bigger number of species are : Asteraceae (151-21.33\%), Poaceae (70-9.89\%), Fabaceae (49$6.92 \%)$, Solanaceae (36-5.08\%) and Malvaceae (29-4.10\%). Of sorts's the total 92 they endemic and they yield 36 in order to the department.

On the basis of the relation among geomorphology, lothology, altitude, floors bioclimaticos, rainfall, description intervals bioclimática, and the composition floristica in given zones, 17 vegetable communities in four very zones put a brand on were differentiated. Predominating the vegetation of hills, tillandsial, riverside mountain, thicket, pajonal, half way deserted, I step on of cactaceae, tolares and queñoales's forests.

\section{INTRODUCCIÓN}

El estudio de la flora vascular y la vegetación del extremo sur del Perú, es una de las zonas con menos información botánica, por lo tanto, con vacío de información. En el departamento de Tacna han sido poco los trabajos realizados y publicados. Haciendo una recopilación sobre los trabajos y colectores que han herborizado en los alrededores de Tacna podemos mencionar a: F.J.Meyen (1831), el naturalista inglés
Pearce (1864), los alemanes G.Woitschach y E. Wedermann (1890 y 1925), Roy Metcalf (1942), realizaron colectas cerca de la ciudad; hasta dicho año todo el material botánico colectado se destruyó en el útimo conflicto bélico. Weberbauer (1945), mercona en uno se sus capitulos a los Andes Occidentales del Sur reportando algunas especies del valle de Candarave y Curibaya; Ferreyra (1961), reporta cerca de 150 especies en las lomas del sur del Perú;

(1) (2) Biólogos.

(3) Bióloga Microbióloga 
Brako \& Zarucchi (1993), menciona 257 especies de Angiospermas y Gimnospermas para el departamento; Dillon (1997), reporta 106 especies en las lomas de Tacna; Zegarra (1992-1996), reporta algunas especies aledañas a la ciudad, Refulio (2000), realizo su trabajo de la familia Poaceae en la Provincia de Tarata, Cáceres \& Franco (1999 al 2003), han intensificado las colectas botánicas por casi todo el departamento por último Galán de Mera \& Cáceres (2002 y 2003), reportan trabajos fitosociologicos del sur peruano.

Con esta información recogida en estos últimos años podemos ya tener un acercamiento sobre la flora vascular y la vegetación del departamento de Tacna, lo más próximo era lo que se conocía en el catalogo de flora Perú con 257 especies y las comunidades mencionadas por Weberbauer (1945). En este trabajo damos a conocer el incremento de la flora para el departamento de Tacna identificando hasta la fecha 708 especies de flora vascular por lo tanto son 451 nuevas adiciones a la flora del Perú y 17 comunidades vegetales en cuatro zonas muy marcadas.

El objetivo de este trabajo es dar a conocer una aproximación de la flora y vegetación del departamento de Tacna,

Precisamente Tacna junto con Moquegua y las partes altas de Arequipa, han sido reconocidas por otros autores como una de las áreas peruanas de menor información Botánica (Cano \& al. 1996).

\section{MATERIAL Y METODO}

\section{AREA DE ESTUDIO}

El departamento de Tacna, se ubica en el extremo sur del Perú enmarcado en al coordenadas geográficas $16^{\circ} 59^{\prime}$ y $18^{\circ} 21^{\prime} 34.8^{\prime \prime}$ LS y $69^{\circ} 28^{\prime}$ y $71^{\circ} 10^{\prime} \mathrm{LW}$, en altitudes que van de los 0.0 a 6000 metros de altitud. Los puntos extremos del territorio tacneño se localizan al norte de la provincia de candarave, frontera con Moquegua, por el Noreste con el departamento de Puno, por el Este con la República de Bolivia, por el sur con la República de Chile, y por el Oeste con el Océano Pacifico. Se divide en cuatro provincias: Tacna, Tarata, Jorge Basadre y Candarave. Vista en toda su extensión, la superficie tacneña presenta dos áreas contratantes. La región de la costa, estrecha, con llanuras ondulantes, cortadas se trecho en trecho por los rios Caplina, Sama y Locumba. torrentosos de pequeño caudal, pero en una persistente proyección hacia el litoral Pacifico. La región andina con relieve accidentado en cuyas altas cumbres se originan las cuencas de los ríos que corren al Pacifico, y la cuenca de los otros, el Maure y el Uchusuma, que, tras breve recorrido, cruzan las fronteras de Chile y Bolivia para internarse en la región altiplánica: destacan, entre las cumbres andinas los volcanes Yucamani (5508 m.) y Tutupaca $(5815 \mathrm{~m}$.) en el distrito de Candarave; los nevados el Frayle (5568 m.) y Barroso (5695 m.) en el distrito de Palca.

\section{COLECCIÓN DEL MATERIAL BOTANICO Y DETERMINACIÓN DE LA VEGETACIÓN}

Las colectas de las plantas y las observaciones se realizaron durante los años de 1998 al 2004, en diferentes estaciones, recorriendo las cuatro provincias del departamento de Tacna (Tarata, Candarave y Jorge Basadre), las áreas de muestreo fueron elegidas por su representatividad en cuanto a la heterogeneidad del paisaje, y por sus posibilidades de ser recorridas. El tipo de muestreo empleado fue el muestreo preferencial o selectivo; donde las muestras o las unidades muestrales son tipicas o representativas sobre la base de criterios subjetivos del autor (Matteucci, 1982). Las muestras colectadas fueron representativas y completas, lo que facilitó su identificación. determinación y clasificación taxonómica de las mismas.

Para ubicar con exactitud las localidades de colecta se emplearon mapas topográficos y un GPS (Sistema de posición global). Se tomaron datos de campo como la localidad, fecha de colecta, altitud, hábitat, forma de vida, datos morfológicos, nombre vulgares. Las técnicas para el procesamiento de las muestras fueron siguiendo a Cerrate (1969).

Para determinar el tipo de vegetación se tuvo en cuenta los trabajos realizados por Weberbauer. Ferreyra y Gaián de Mera \& Cáceres.

\section{DETERMINACIÓN DEL MATERIAL. BOTANICO}

La identificación y determinación se realizó haciendo uso de bibliografia especializada, investigadores especialistas y mediante la comparación de las muestras colectadas con los ejemplares existentes en el Herbario San Marcos del Museo de Historia Natural de la Universidad Nacional Mayor de San Marcos. Para la clasificación taxonómica se utilizó: Tryon \&. Tryon (1982), para las Pteridophyta; Raven (1999), para las Gnetophyta y Cronquist (1981), para las Magnoliophyta.

\section{V.RESULTADOS}

Se identificaron 708 especies, 360 géneros, 101 familias y 4 divisiones, distribuidas en: Pteridophytas (21 especies, representan el $2.97 \%$ ), Coniferophyta (3$0.42 \%)$. Gnetophyta (2- $0.28 \%$ ) y Magnoliophyta (Magnoliopsida, 580-81. 92\% y Liliopsida, 102 $14.41 \%$ ). Las familias con mayor número de especies son: Asteraceae(151-21.33\%), Poaceae (70-9.89\%), 
Fabaceae (49-6.92\%), Solanaceae (36-5.08\%) y Malvaceae (29-4.10\%). Entre las formas biológicas más dominantes tenemos a las hierbas seguidas de los arbustos y por último a los árboles. Del total de las especies 92 son endémicas y 36 solo se reportan para el departamento.

Para determinar las comunidades vegetales se analizaron los datos de registrados desde 1998 al 2004 , de acuerdo a las investigaciones de campo y ha estudios propuestos por diferentes autores, como resultado se presenta a continuación las diferentes comunidades en cuatro zonas muy marcadas.

En la costa: Humedales; con especies representativas de Typha dominguesis, Bacopa monnieri y Distichlis spicata. Lomas; Palaua dissecta, Nolana arenicola, N. adansonii, N.confinis, N.lycioides, Tiquilia paronychioides, Loasa urens, Salvia rhombifolia, Cotula australis, Carica candicans, Cleome chilensis. Tillandsial; Tillandsia wedermanii y Tillandsia purpurea. Monte ribereño, Acacia macracanta, Tessaria integrifolia, Schinus molle, Arundo donax, Prosopis chilensis y Leucaena leucocephala. Formaciones de cactaceas; Neoraymondia arequipensis, Neoporteria islayensis y Corryocactus brachypetalus. Formaciones de arbustos perenifolios; Tecoma fulva, Pluchea chingoyo y Grindelia glutinosa. Relictos de chañal; Geoffroea decorticans.

En la Sierra: Formaciones de cactaceas; Browningia candelaris, Haageocereus australis, Corryocactus brevistylus y Oreocereus leucotrichus. Puyales; Puya aff angularis. Matorral; Baccharis tricuneata, Baccharis boliviensis, Proustia beberidifolia, Balbisia meyeniana, Ophryosporus peruvianus, Diplostephium meyenii, Dunalia spinosa, Junellia arequipensis, Grindelia tarapacana, Tarasa operculata, Ambrosia artemisioides. Monte ribereño; Tessaria integrifolia, Muehlenbeckia hastulata, Otholobium pubescens, Cortaderia jubaba, Cantua buxifolia, Colletia spinosa, Hydrocotyle bonariensis, Rorippa nasturtium-aquaticum, Lycopersicon chilense, Nicotiana glauca, Baccharis petiolata.

En la Puna: Matorrales; Parastrephia lepidophylla, P.lucida, Chersodoma jodopappa, Adesmia spinosissima, Tetraglochin cristatum, Baccharis tricuneata, Junellia juniperina y frazales de Fabiana stephanii. Pajonal; Festuca orthophylla y Stipa ichu. Queñoales, Polylepis besseri, Polylepis tarapacana, Chuquiraga rotundifolia, Calceolaria inamoena, Bomarea dulcis. Turberas y bofedales; Plantago tubulosa, Distichia muscoides, Werneria pygmaea, Calamagrostis ovata, Hypsela reniformes, Oxychloe andina y Yaretales; Azorella compacta, Valeriana nivalis, Bartsia peruviana.

En la zona Subnival: Formaciones almohadillados: Pycnophyllum molle. Semidesierto: Nototriche argentea, N. foetida, N. alternata, Astragalus peruvianus, Xenophyllum lycopodioides $\mathrm{X}$. poposum.

\section{DISCUSIÓN}

Con este trabajo de investigación se pone de manifiesto una aproximación sobre la flora vascular del departamento de Tacna. Se cita por primera vez 708 especies de la flora vascular para este departamento, siendo 92 endémicas y 36 solo registradas para el departamento de Tacna; se realiza por primera vez 451 adiciones a la flora de Perú.

Las familias mejor representadas son las Asteraceae(151-21.33\%), Poaceae (70-9.89\%), Fabaceae (49-6.92\%), Solanaceae $(36-5.08 \%)$ y Malvaceae $(29-4.10 \%)$, se demuestra que las Asteraceas es una de las familias mas representativas de las zonas andinas de nuestro territorio peruano. Entre las especies endémicas podemos mencionar a Domeykoa saniculifolia y Eremocharis confinis (Apiaceae); Chaetanthera peruviana, Chersodoma arequipenis, Ch.diclina, Ch.juanisemii,Diplostephium meyenii, Heterosperma ferreyrii, Philoglosa peruviana, Senecio adenophyllus, Senecio modestus, Senecio trifurcifolius, Senecio candolli, Viguiera weberbaueri, Werneria ciliolata, Werneria caespitosa, Werneria strigosissima (Asteraceae); Pectocarpa laterioflora, Tiquilia elongata, Tiquilia tacnenis, (Boraginaceae); Tillandsia latifolia y Tillandsia wedermanii, (Bromeliaceae); Cleistocactus sextonianus, Corryocactus brachypetalus, Haageocereus plantinospinus, Neoraimondia arequipensis, Neoporteria islayensis, Opuntia pyrrhacantha, Opuntia corotilla, Oreocereus tacnaensis, Trichocereus tacnaensis (Cactaceae); Drymaria fasciculata Spergularia collina, (Caryophyliaceae); Carica candicans (Caricaceae); Villadia reniformes (Crassulaceae); Euphorbia tacnensis (Euphorbiaceae); Adesmia augusti, Dalea pennellii, Dalea exilis, Lupinus exochus, L.ericladua, L.paruroensis, L.pinguis, proculaustrinus, Weberbauerella bronginiartioides, (Fabaceae); Escallonia salicifolia (Grossulariaceae); Cardenanthus peruvianus, Trigidia albicans, (Inidaceae); Antherium glaucum, Bomarea tacnaense (Liliaceae); Caiophora pentlandii, C.sepiaria, C.stenocarpa, (Loasaceae); Malesherbia turbinea (Malesherbiaceae); Nototriche borussica, N.foetida, Palaua velutina, Tarasa tenuis Urocarpidium albiflorum, (Malvaceae); Nolana spp (Nolanaceae); Oxalis lomana (Oxalidaceae); Plantago limensis, PI.serica, PI.tacnensis, (Plantaginaceae); Dissanthelium macusaniense, Eragrostis weberbaueri, Poa aequigluma, Stipa rigidiseta, (Poaceae); Cantua cadelilla, (Polemoniaceae); Monnina macbridei, (Polygalaceae); Calandrina acaulis, Cistanthe paniculada, Portulaca nivea, (Portulacaceae); Leptoglossis darcyana, Nicotiana glutinosa, Solanum acroscopium, 
En niranto a la veqetación existente en la Costa se presentan las Lomas, siendo una formación vegetal de la costa desértica del pacífico sur, las lomas han sido estudiadas por algunos investigadores como Ferreyra (1961) y Dillon (1997), en estas formaciones son tipica las hierbas como Nolana spp, Palaua spp, Tetragonia spp, entre otras. El Tillandsial, vegetación de las zonas áridas de la costa peruana representado por Tillandsia spp, siendo $\mathrm{T}$. wedermanni registrado sólo para el departamento de Tacna. El Monte Ribereño lo característico alli a lo largo de las tres cuencas importante de Tacna crecen especies representativas como Schinus molle, Tecoma fulva, Pluchea chingollo, Acacia spp, Tessaria integrifolia. El Chañal, es un relicto de un bosque de Geofroea decorticans, que se ubica en la costa tacneña. El Desierto Costero, ofrece planicies extensas con poca o nula precipitación.

En las Vertiente Occidentales encontramos el Piso de Cactaceas caracterizado por Browningia candelaria, Corryocactus brevistylus, Haageocereus spp, Opuntia corotilla. El Matorral, una zona de vegetación donde se puede apreciar que predominan los arbustos medianos como Baccharis spp, Diplosthephium spp, Cantua buxifolia, Colletia spinosa, Grindelia tarapacana, Ophryosporus peruvianus, Junellia arequipense, Viguiera procumbens, Proustia berberidifolia, entre otras.

La Puna Tacneña esta representada por vegetaciones típicas como los Tolares, predominado por Parastrepia quadrangularis, Parastrephia lucida, Baccharis tricuneata; Estepa arbustiva representados por Adesmia spinosissima, Tetraglochin cristatum, Junellia juniperiana; Frazales de Fabiana. son comunidades típicas de Fabiana stephanii y Baccharis boliviensis ; Los Yaretales predominado por Azorella compacta, Valeriana nivalis, Woodsia montevidensis, Salpichroa glandulosa. Los Pajonales, cubren grandes extensiones de territorio son muy comunes Festuca spp, Stipa spp y Anthochloa spp. Los Bosque de Polylepis, son formaciones característicos de algunas zonas altoandinas represntado por Polylepis besseri y Polylepis tarapacana y la Vegetación Subnival caracterizado por especies bajas al ras del sueio entre ellas Pycnophyllum molle, Nototriche spp y Werneria spp.

\section{CONCLUSIONES}

Se registra un total de 708 especies, comprendidas en 101 familias $\vee 4$ divisiones

Se distribuyen en: Pteridophytas ( 21 especies, representan el $2.97 \%$ ), Coniferophyta $(3-0.42 \%)$, Gnetophyta (2- $0.28 \%$ ) y Magnoliophyta ( Magnoliopsida, 580-81. 92\% y Liliopsida, 102-
Las iamillas con miayui huiticiv ue espevies oun. Asteraceae(151-21.33\%). Poaceae $(70-9.89 \%)$. Fabaceae $(49-6.92 \%)$. Solanaceae $(36-5.08 \%)$ v Malvaceae (29-4 10\%)

Entre las formas biológicas más dominantes tenemos a las hierbas seguidas de los arbustos y por último a los árboles. Del total de las especies 92 son endémicas y 36 solo se reportan para el departamento.

Fueron determindas 17 comunidades vegetales en cuatro zonas muy marcadas. Predominando la vegetación de lomas, tillandsial, monte ribereño, matorral, pajonal, semi desierto, piso de cactaceas, tolares y bosques de queñoales.

\section{FLORAY VEGETACIÓN DEL DEPARTAMENTO DE TACNA}
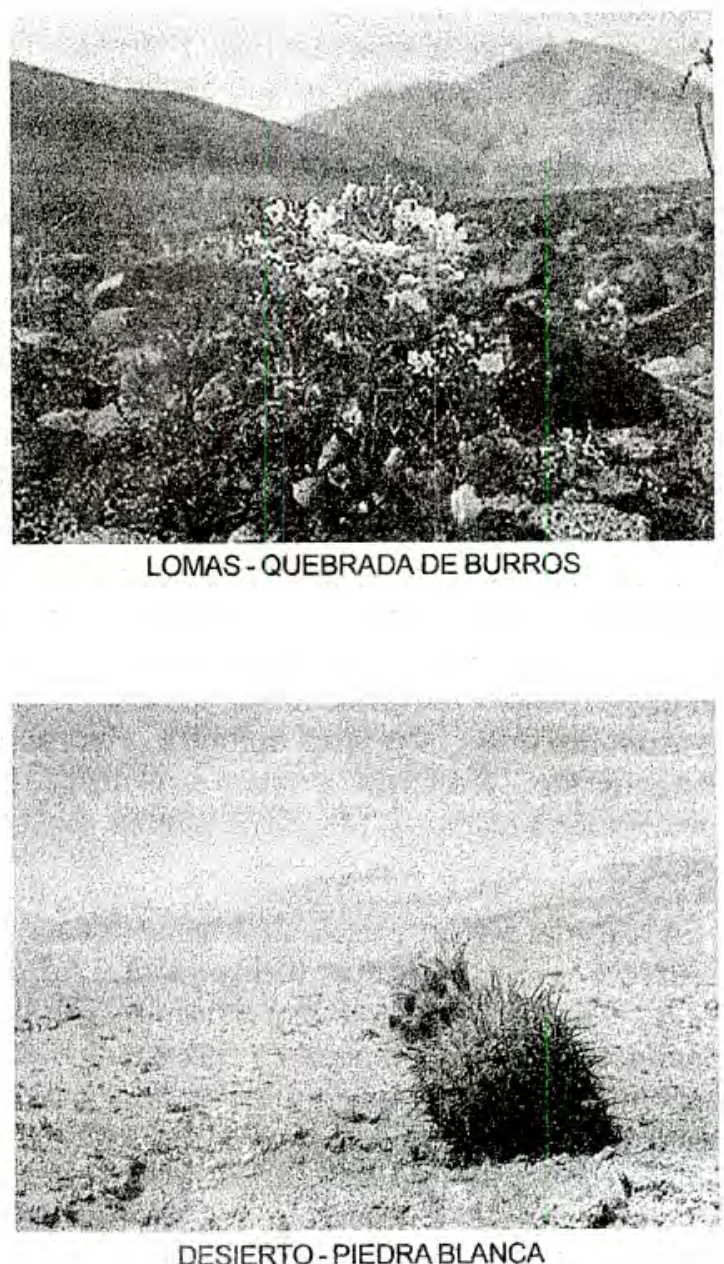

DESIERTO-PIEDRABLANCA 


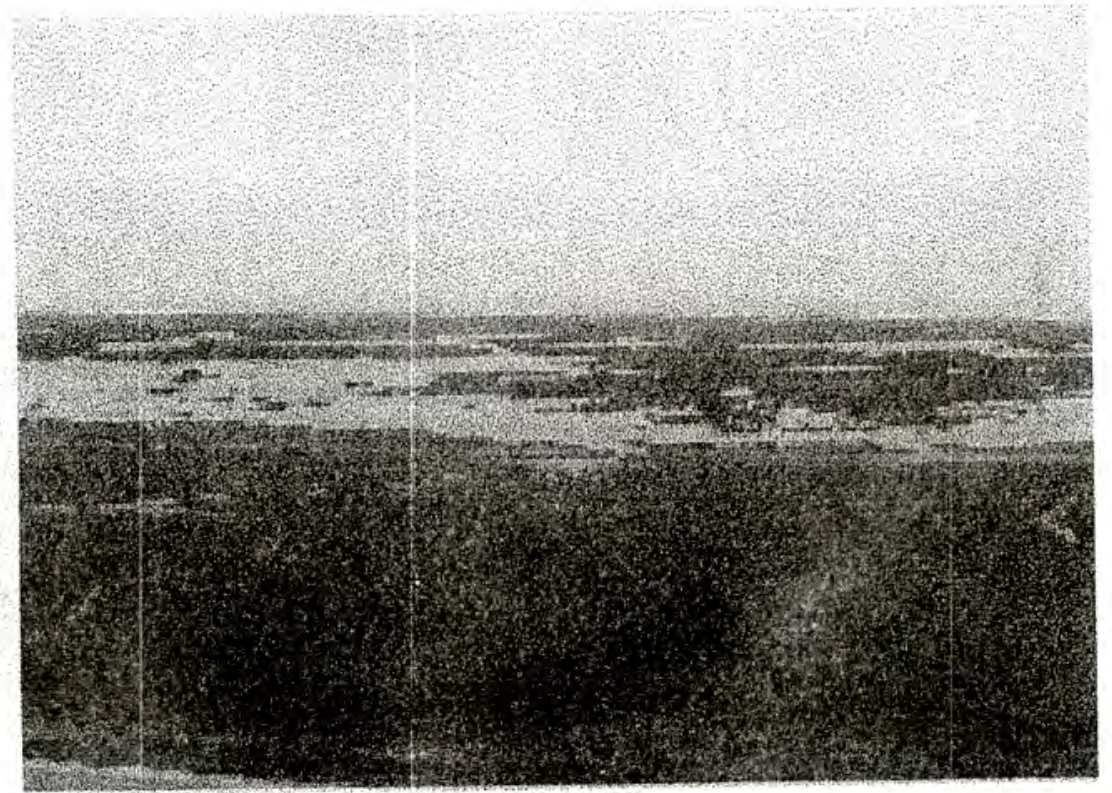

HUMEDAL-ITE
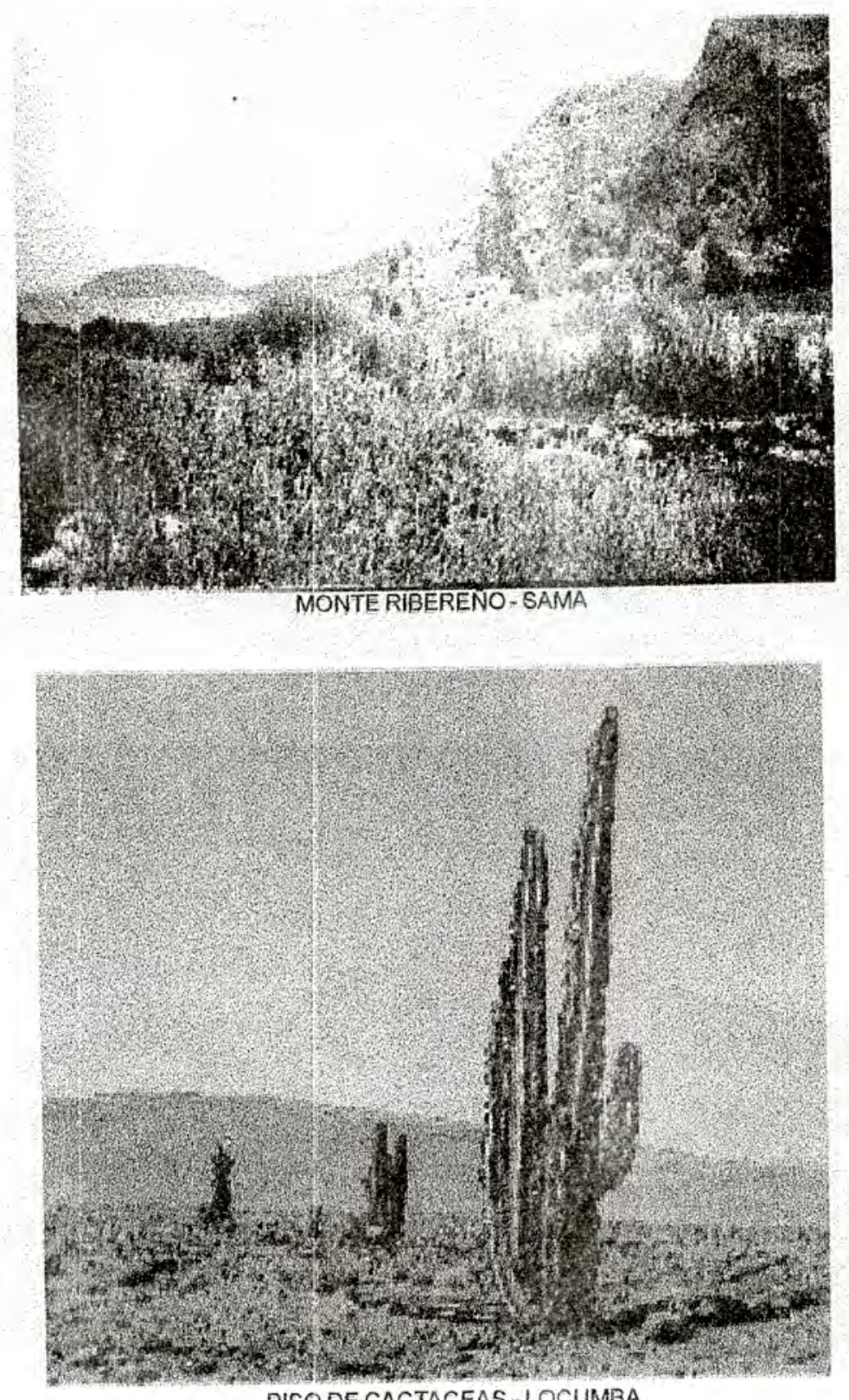

PISO DE CACTACEAS - LOCUMBA 


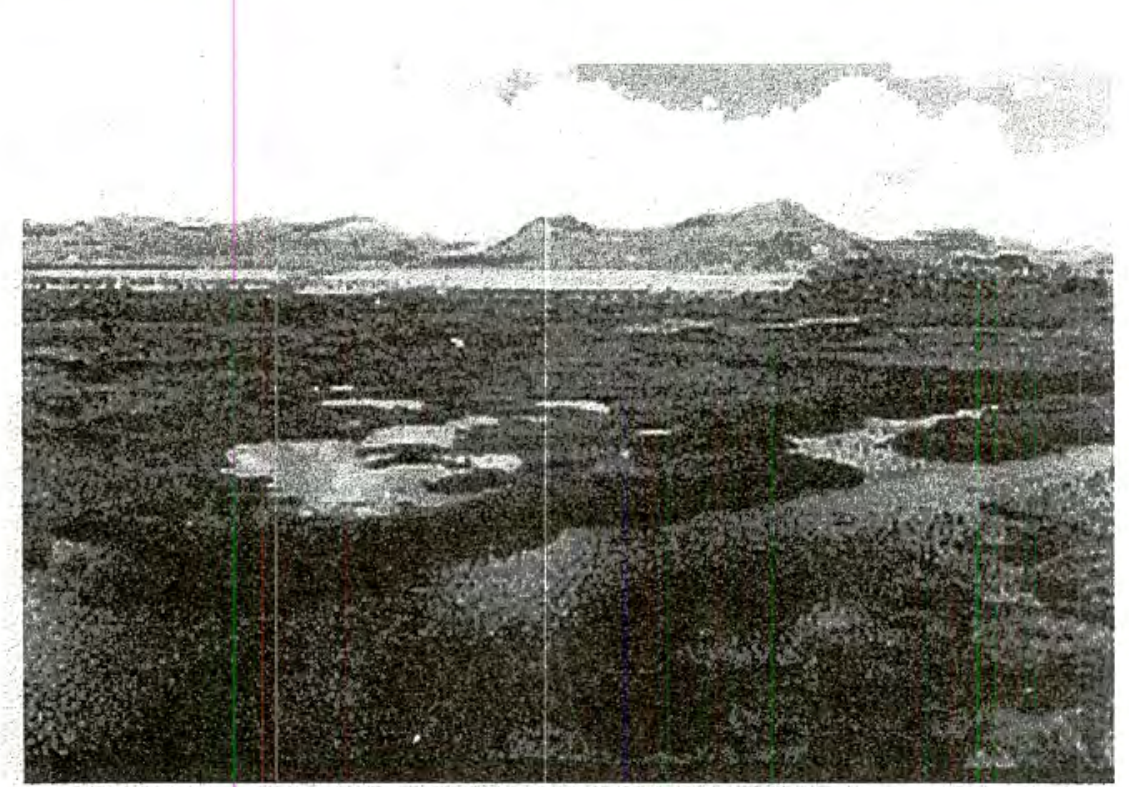

BOFEDAL-TRIPARTITO

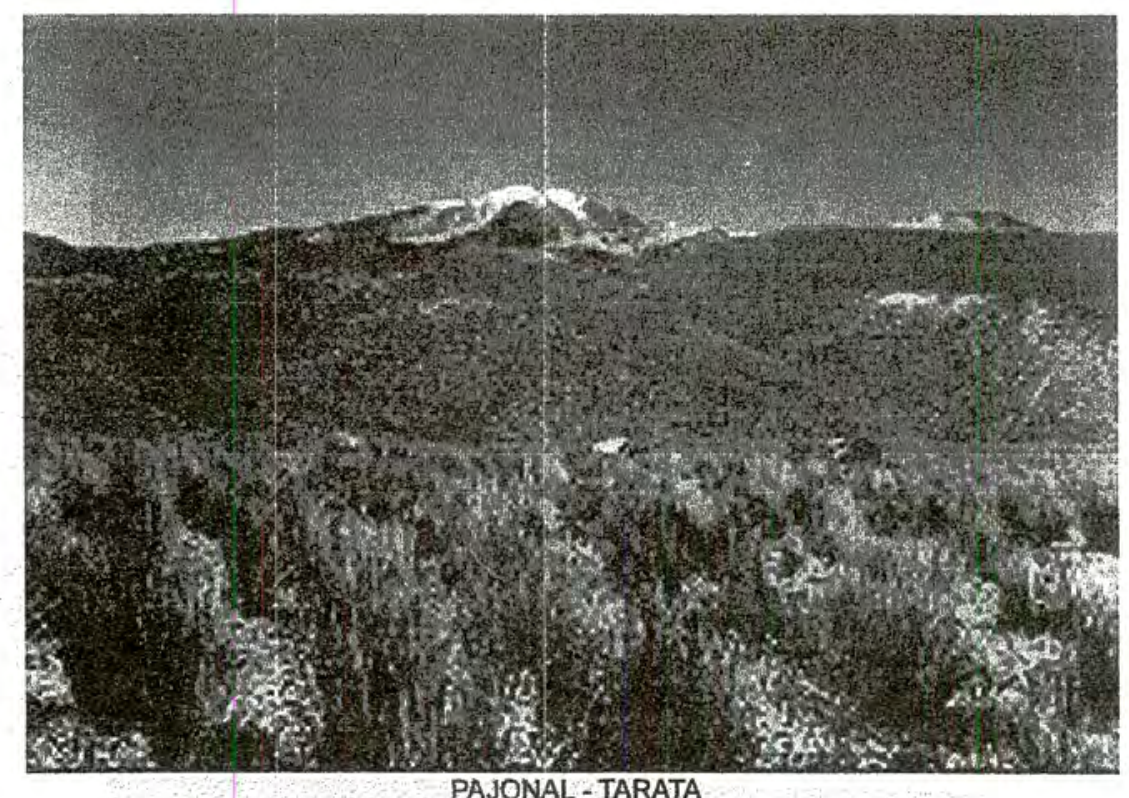

PAJONAL - TARATA

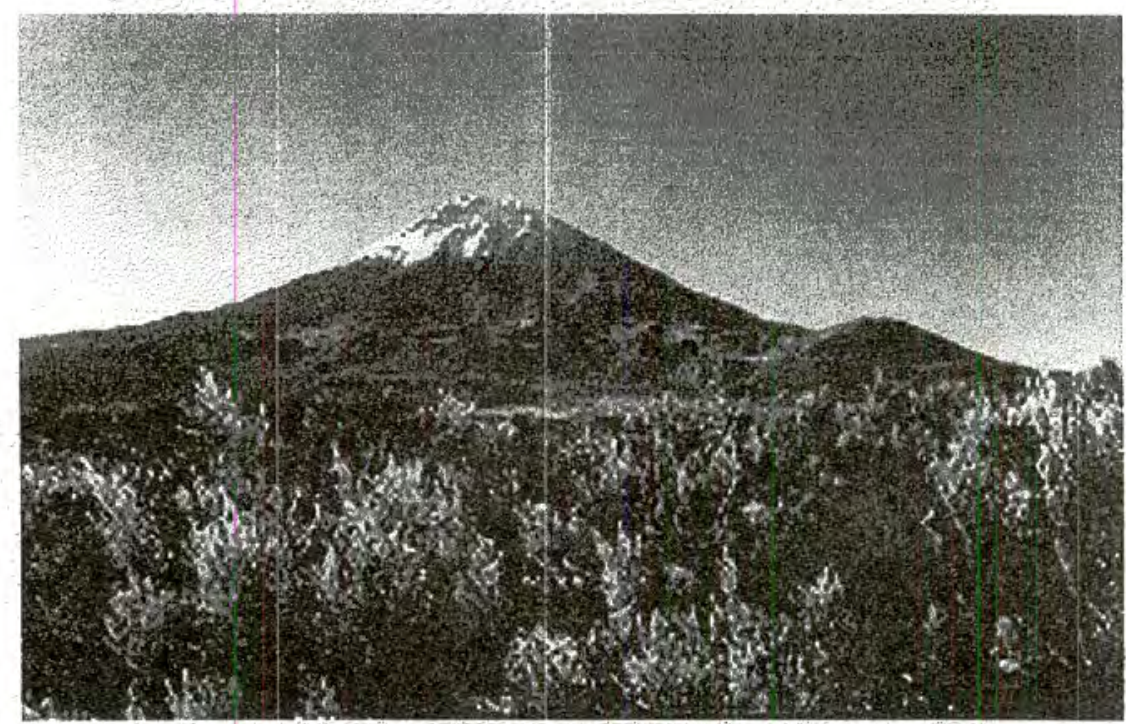

TOLAR - CANDARAVE 

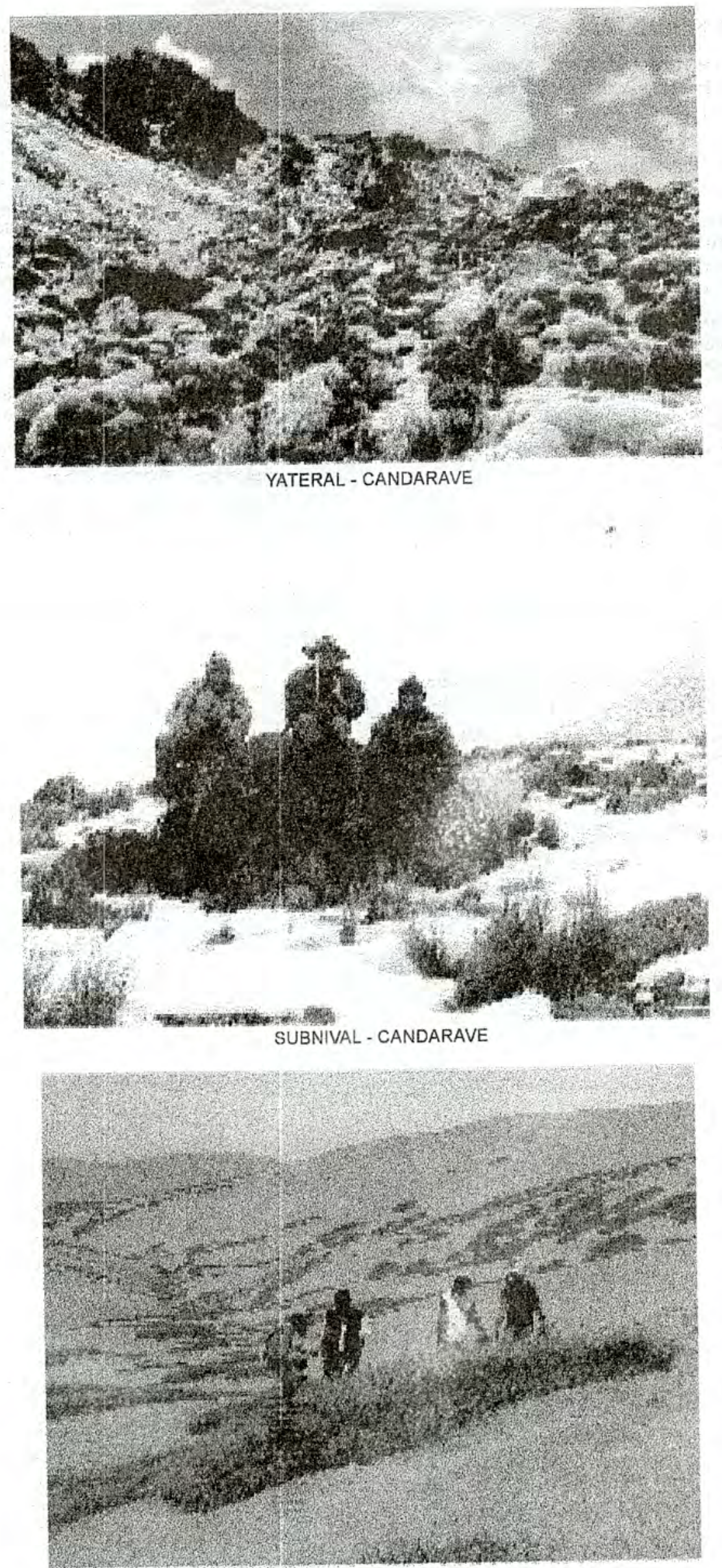

TILLANDSIAL - TACNA 


\section{REFERENCIA BIBLIOGRÁFICA}

- BRAKO, L. \& ZARUCCHI, J. 1993. Catálogo de las angiospermas y gimnospermas del Perú.Missouri botanical garden. 1286 pag.

- CACERES, C. \& FRANCO, J. 2000. Plantas medicinales del distrito de palca. VIII Congreso Nacional de Botánica. Arequipa-Perú

- CACERES, C. \& FRANCO, J. 2001. Datos etnobotánicos de la comunidad de Viltavira. Provincia de Candarave,Tacna. X Reunión cientifica. Instituto de ciencias biolóbicas Antonio Raimondi. Lima-Perú.

- CACERES, C. \& FRANCO, J. 2002. 'Estado Actual del Tillansial en el departamento de Tacna. XI Reunión cientifica. Instituto de ciencias biolóbicas Antonio Raimondi. Lima-Perú.

- CACERES, C. \& FRANCO, J. 2003. La tribu Senecionae( Ásteraceae) en el departamento de Tacna. XII Reunión científica. Instituto de ciencias biológicas Antonio Raimondi. LimaPerú.

- CACERES, C. \& FRANCO, J. 2004. Asteraceas comunes de la Provincia de Candarave-Departamento de Tacna y su importancia como recurso natural. Revista Ciencia y Desarrollo-Universidad Nacional Jorge Basadre Grohmann.

- CANO, A \& COL. 1996. Areas importantes para la conservación de fanerógamas en el Perú.Proyecto FANPEGZT-INRENA-Lima-Perú

- CERRATE, E. 1969. Manera de preparar plantas para un herbario.Museo de Historia Natural. UNMSM.Lima-Perú.

- FERREYRA, R. 1961. Las lomas costeras del extremo sur del Perú. Boletín de la Sociedad Argentina de Botánica.Buenos Aires-Argentina.
FERREYRA, R. 1983. Los tipos de vegetación de la costa peruana. Anales del Jardín Botánico de Madrid- Españe.

- GALAN, M. \& CACERES,C. 2002. Una aproximación sintaxonómica sobre la vegetación del Perú. Clases,Ordenes y Alianzas. Revista Acta Botánica MalacitanaEspaña.

- GALAN, M. \& CACERES,C. 2002. Las comunidades con cactáceas del sur del Perú. nueva asociación v alianza del desierto Pacífico. Revista Acta Botánica Malacitana-España.

- GALAN, M. \& CACERES,C. 2003. La Vegetación de la alta montaña andina del Sur del Perú. Revista Acta Botánica Malacitana-Espan̉a

- GALAN, M. \& CACERES,C. 2003. Nuevas adiciones a la flora del Perú, 111. Revista Candollea-Suiza.

- MATTEUCCI, S. 1982. Metodología para el estudio de la vegetación. Organización de los estados americanos. OEA.

- RAVEN, P. 1999. Biology of plant. 6ta edition. Freeman \& Company.Worth. Publisbers. New York.

- TRYON, R \& TRYON, A. 1982. Ferns and allied plants, with special refernce to tropical america springer. New York.

- WEBERBAUER, A. 1945. El mundo vegetal de los Andes peruanos. Estudio Fitogeográfico. Estación experimental agricola de la Molina. Dirección de Agricultura. Lima-Perú. 